\title{
BIENESTAR ECONÓMICO: UNA APROXIMACIÓN ANALÍTICA Y CRÍTICA ${ }^{1}$
}

\author{
ECONOMIC WELFARE: AN ANALYTICAL AND CRITICAL APPROACH
}

\section{BEM-ESTAR ECONÔMICO: UMA ABORDAGEM ANALÍTICA E CRÍTICA}

\author{
RIASCOS HERMOZA_Julio César, ACOSTA PANTOJA_Luisa Fernanda, \\ ERAZO ANDRADE_ Iván Fredy
}

Magíster en Gerencia y Asesoría Financiera, Universidad Mariana. Docente e investigador Universidad Mariana y Universidad de Nariño. E-mail: jriascos2009@ hotmail.com, Colombia.

Especialista en Finanzas, Universidad de Nariño. Integrante del Grupo de Investigación Desarrollo Regional (IDER) de la Universidad de Nariño. E-mail: Lufeacosta94@ gmail.com, Colombia.

Magíster en Gerencia y Asesoría Financiera, Universidad Mariana. Docente e investigador de la Universidad de Nariño. E-mail: ivanerazo59@hotmail.com, Colombia.

Recibido: 1 de octubre de 2019

Aprobado: 30 de marzo de 2020

DOI: https://doi.org/10.22267/rtend.202101.132

\section{RESUMEN}

El propósito central del presente artículo radica en efectuar una consideración analítica sobre las tesis asociadas a la teoría del bienestar desarrolladas por autores como Bentham (1786), Edgewoth (1881), Arrow (1974), Sen (2000), Max-Neef (2011) y Deaton (2015). Por medio de una revisión

\footnotetext{
${ }^{1}$ Este artículo es producto de las investigaciones tituladas "Incidencia de los Programas de Sistema de Bienestar Universitario Sobre la Situación de Pobreza Monetaria de los Estudiantes de Pregrado de la Universidad de Nariño, Sede Pasto. 2018" llevado a cabo por los Grupos de Investigación de Desarrollo Regional (IDER) y Coyuntura Económica y Social (CES) en la Universidad de Nariño, Colombia.
} 
documental basada en obras y artículos científicos originales, se examinó el concepto de bienestar e importancia en la teoría económica. El documento aborda de una parte, el enfoque ortodoxo fundamentado en el utilitarismo, precisando la elección individual de bienes y servicios como instrumento que posibilita la satisfacción de necesidades y, por ende la maximización de su situación de bienestar; de otro lado, el paper analiza la teoría de las funciones y capacidades o, también denominada de libertades fundamentales, que exalta la heterogeneidad del ser humano y sus condiciones de vida objetivamente. La principal conclusión de este documento estriba en que el concepto de bienestar está basado en dos posturas claramente diferenciadas, una primera que sugiere que el concepto puede ser modelado en una función de utilidad, y un segundo enfoque que promueve que se trata de un término complejo pues está compuesto de expresiones multidimensionales de desarrollo.

Palabras clave: bienestar, utilidad, teoría económica.

JEL: D6, I3, I31

\begin{abstract}
The central purpose of this article is to make an analytical consideration of the theses associated with the theory of welfare developed by authors such as Bentham (1786), Edgewoth (1881), Arrow (1974), Sen (2000), Max-Neef (2011) and Deaton (2015). Through a documentary review based on original scientific works and articles, the concept of welfare and importance in economic theory was examined. The document addresses, on the one hand, the orthodox approach based on utilitarianism, specifying the individual choice of goods and services as an instrument that enables the satisfaction of needs and, therefore, the maximization of their welfare situation; On the other hand, the paper analyzes the theory of functions and capacities or, also called fundamental liberties, which exalts the heterogeneity of the human being and his living conditions objectively. The main conclusion of this document is that the concept of welfare is based on two clearly differentiated positions, a first that suggests that the concept can be modeled in a utility function, and a second approach that promotes that it is a complex term, it is made up of multidimensional expressions of development.
\end{abstract}

Keywords: welfare, utility, economic theory.

JEL: D6, I3, I31 


\section{RESUMO}

O objetivo central deste artigo é fazer uma análise analítica das teses associadas à teoria do bemestar desenvolvida por autores como Bentham (1786), Edgewoth (1881), Arrow (1974), Sen (2000), Max-Neef (2011) e Deaton (2015). Através de uma revisão documental baseada em trabalhos e artigos científicos originais, foi examinado o conceito de bem-estar e importância na teoria econômica. O documento aborda, por um lado, a abordagem ortodoxa baseada no utilitarismo, especificando a escolha individual de bens e serviços como um instrumento que possibilita a satisfação de necessidades e, portanto, a maximização de sua situação de bem-estar; por outro lado, o artigo analisa a teoria das funções e capacidades ou, também chamadas liberdades fundamentais, que exaltam a heterogeneidade do ser humano e suas condições de vida objetivamente. A principal conclusão deste documento é que o conceito de bem-estar se baseia em duas posições claramente diferenciadas, uma primeira que sugere que o conceito pode ser modelado em uma função de utilidade e uma segunda abordagem que promove que é um termo complexo. é composto de expressões multidimensionais de desenvolvimento.

Palavras-chave: Bem-estar, utilidade, teoria econômica.

JEL: D6, I3, I31

\section{INTRODUCCIÓN}

"Si encuentras modo de proporcionar a los que han de mandar una vida mejor que la del gobernante, es posible que llegues a tener una ciudad bien gobernada, pues esta será la única en que manden los verdaderos ricos, que no lo son en oro, sino en lo que hay que poseer en abundancia para ser feliz: una vida buena y juiciosa. Pero donde son mendigos y hambrientos de bienes personales los que van a la política creyendo que es de ahí de donde hay que sacar las riquezas, allí no ocurrirá así. Porque cuando el mando se convierte en objeto de luchas, esa misma guerra doméstica e intestina los pierde tanto a ellos como al resto de la ciudad."

Platón (La República).

Uno de los factores claves de la ciencia económica radica en la comprensión del bienestar como producto de un sistema productivo y social, no obstante, resulta relevante determinar ¿Cuál es la visión con que el concepto de bienestar ha sido tratado dentro de la teoría económica subyacente? 
A menudo los economistas discuten sobre el eje conceptual y metodológico con que la ciencia se debe estudiar, como respuesta han surgido diversas escuelas del pensamiento que invitan a reflexionar sobre cada problemática o fenómeno desde diferentes perspectivas, algunas siendo más aclamadas que otras. Inicialmente, economistas como Adam Smith, David Ricardo, Thomas Malthus, Jean Baptiste-Say y François Quesnay, concentraron su atención en tópicos como la oferta, la demanda, el valor, la libre competencia, el trabajo, la renta y la producción, que eran ajustados de acuerdo a su contexto temporal y espacial respectivo.

Con posteridad, devino como reacción la perspectiva de Karl Marx, cuyo concepto de plusvalía y el engranaje económico y social que le soportaba, determinó un impulso trascedente al sector obrero para la creación de sindicatos, como consecuencia de las precarias condiciones de trabajo de su época.

Seguidamente, los axiomas desarrollados por la escuela neoclásica, sirvieron de base para el desarrollo del análisis económico moderno, haciendo énfasis en el subjetivismo y la pretensión de comprender la psicología humana en relación a sus gustos, preferencias, utilidad y satisfacción percibida con el consumo de bienes y servicios, además de incorporar un rol particular al capital especulativo para la obtención de riqueza y crecimiento. La crisis contraída con la aplicación de tales ideas, fue contrarrestada por John Maynard Keynes y su propuesta de un Estado intermitente en la dinámica económica.

Por otro lado, la escuela neoclásica supuso que el bienestar humano dependía, en buena medida, del crecimiento económico, entendido como la capacidad manifiesta de producir un mayor volumen de bienes y servicios para ofrecer satisfacción a los agentes que componen a la economía. A su turno, teorías de corte social comprenden una definición menos convencional y por tanto, más desafiante, porque no solo conciben al bienestar a partir de las carencias de un ingreso suficiente, de las dificultades de un proceso inflacionario, de las escasas oportunidades para acceder a un sistema de salud o a los servicios de educación, sino que se adhieren a la interrelación de factores complejos propios del tejido social subyacente, la compleja dinámica humana y la búsqueda de las preferencias valorativas que delimiten su desarrollo.

A lo largo del presente estudio se efectuó una revisión analítica de la literatura existente por medio de la cual, se pretendió reconocer el conjunto de posturas teóricas sobre el bienestar, desarrolladas 
por autores reconocidos y merecedores de Premios Nobel de Economía. En este sentido, documento se compone de tres apartados que datan sobre la temática desde una mirada de política pública, el enfoque del utilitarismo y la visión otorgada por Sen y Deaton, culminando con unas conclusiones teóricas y conceptuales.

\section{REFERENTES TEÓRICOS}

El bienestar en la teoría económica, ha incorporado una multiplicidad de elementos que articulan y nutren el debate entre las diferentes escuelas que pretenden estudiarla; en este sentido, Arrow (1951) mediante su teorema de la imposibilidad, hace visible la importancia de fundamentar su análisis de las elecciones de los individuos sobre sus valores y no en la simpleza de sus gustos, por lo cual, manifiesta que el bienestar no se logra a través de la dinámica ejercida en el mercado como lo ha considerado la teoría ortodoxa liderada por Smith, Bentham y Mill a través del utilitarismo, puesto que este carece de elementos inherentes a la complejidad y multidimensionalidad de las necesidades y aspiraciones del hombre. En base a la teoría de Arrow, González (1998) advierte lo siguiente:

Pensar que la función de bienestar social se deriva de las fuerzas autónomas del mercado es negar la complejidad inherente al proceso de elección social. El discurso neoliberal repite, una y otra vez, que la eficiencia del mercado conduce al bienestar general. Esta posición prekantiana no es más que una ideologización del mercado (González, 1998: 36).

Por otro lado, Deaton, Sen y Max-Neef pretenden conceder al bienestar un enfoque que adiciona instancias que no solo dependen del accionar del mercado, dado que agregan variables que proporcionan un análisis integral del mismo, visto desde las funciones, capacidades, y factores macro y micro complejos, donde intervienen la salud, la esperanza de vida, la educación, calidad de vida, entre otros.

\section{METODOLOGÍA}

La literatura sistemática permite identificar, evaluar e interpretar investigaciones documentales con el objeto de dar respuesta o afianzar las tesis a que haya lugar (Kitchenham, 2004); en este caso particular el examen analítico que da soporte al concepto de bienestar, a través del diagnóstico de los estudios individuales efectuados por los autores en la materia. 
Considerando que la panorámica teórica del bienestar es extensa, esta revisión documental se circunscribió a las obras de autores representativos, algunos de ellos galardonados con el premio Nobel en Economía, puesto que han aportado conceptualizaciones significativas respecto a esta materia.

Con ese propósito, el documento aborda al concepto de bienestar económico, visto desde ópticas como el utilitarismo, con exponentes como Bentham y Arrow; o el ámbito social representado por Sen y Deaton, que posibilitan espacios de crítica y contribución analítica que enriquecen al aporte científico en la economía.

\section{DISCUSIÓN}

\section{Bienestar y Política Pública}

Uno de los desafíos en el diseño e implementación de política pública reside en la dicotomía suscitada entre quienes resultan beneficiados en su calidad de vida y aquellos que no. Platón en la República, declara que la tarea del gobernante, más allá de procurarse su propio bienestar, buscaba asilo en posibilitar a los ciudadanos una mejor calidad de vida; no obstante, la historia del pensamiento económico ha debatido intensamente sobre los medios y las formas de organización social que hacen posible un mejor desarrollo humano. En el mercantilismo, pensadores como William Petty (1899) consideraban que la sociedad se constituía en un organismo con una complejidad semejante a la anatomía humana, pues siendo médico, pronto advirtió que el tejido social y económico exhibía patologías diversas y demandaba sus propios cuidados.

En su tratado intitulado “The Economic writing” de 1899, argumentó que el Estado debía ocuparse de actividades como la administración de justicia y defensa, además de labores de naturaleza social como el financiamiento de instituciones educativas, hospitales e infraestructura, para lo cual se debía recurrir al cobro de impuestos. Lo anterior, dentro de una visión parsimoniosa que no desalentara las iniciativas del sector privado. En este sentido, la política pública requería identificar, por un lado, el conjunto de necesidades sociales como una expresión global de lo que cada individuo precisaba; y, de otra parte, los recursos para desarrollar las tareas del sector público que se relacionaban con gravar una parte de los ingresos. 
En la Teoría de los sentimientos morales (1759) e Investigación sobre la naturaleza y causas de la riqueza de las naciones (1776), Smith propone que el estudio de la riqueza y su principal determinante encabezado por la división del trabajo es relevante dado que, permite un mayor grado de bienestar a la sociedad en su conjunto. Aun cuando los individuos que forman parte de ella actuasen de forma egoísta, la interrelación de los mismos dentro del proceso productivo y las necesidades de intercambio que demandaba la economía, conducían a productores y consumidores a alcanzar por medio de una "mano invisible" el mayor nivel de bienestar colectivo a través del mercado $^{2}$.

A pesar de que la teoría de Smith (1776), inicialmente supone que el mercado constituye un mecanismo conveniente para alcanzar el bienestar colectivo al que aspira una determinada sociedad, y, en consecuencia, establece un radio de acción limitado para el desarrollo de la política pública, el propio autor dedica el libro V de la Riqueza de las Naciones al estudio del Estado y sus funciones.

Hay casos en que la situación misma de la sociedad coloca a la mayor parte de los individuos en condiciones de adquirir por su cuenta, sin la intervención del Gobierno, todas aquellas técnicas y virtudes que el Estado exige o admite. En otras circunstancias, la sociedad no coloca a la mayor parte de los individuos en semejantes condiciones, y entonces es necesaria la atención del Gobierno para precaver una entera corrupción o degeneración en la gran masa del pueblo (Smith, 1776: 687).

\footnotetext{
${ }^{2}$ En el capítulo II de la Riqueza de las Naciones Smith (1776: 17) señala que las necesidades exhibidas por las personas dentro de una sociedad, dependen más de una conducta egoísta que de una motivación benevolente. La frase "Pero el hombre reclama en la mayor parte de las circunstancias la ayuda de sus semejantes y en vano puede esperarla solo de su benevolencia", sugiere en todo caso, que el autor no adopta una posición extrema que pudiese ser interpretada como que dicha ayuda esperada por otros individuos, entonces dependa solamente del egoísmo.

En una lectura complementaria al primer capítulo de la teoría de los sentimientos morales, permite evidenciar que Smith (1759) no niega una combinación de conductas entre el egoísmo y la benevolencia en los seres humanos, aunque con una tendencia hacia la primera sin abandonar totalmente la segunda, pues expresa que:
}

Por más egoísta que quiera suponerse al hombre, evidentemente hay algunos elementos en su naturaleza que lo hacen interesarse en la suerte de los otros de tal modo, que la felicidad de estos le es necesaria, aunque de ello nada obtenga, a no ser el placer de presenciarla. (Smith, 1759: 31).

De hecho, el padre de la economía advierte que, el comportamiento humano se soporta en seis motivaciones a saber: el egoísmo, la conmiseración, el deseo de libertad, el sentido de propiedad, el hábito de trabajar y la propensión al intercambio de bienes y servicios.

Suponer que los fundamentos clásicos de la economía planteados por Smith se basan únicamente en el egoísmo constituye una interpretación radical de la complejidad de su pensamiento. Es necesario considerar este aspecto porque, como se planteará en Arrow, la construcción de una política pública parte del entendimiento de conductas individuales que precisan un ordenamiento global para analizar la posibilidad del bienestar conjunto. 
Para Smith los frutos de la división del trabajo que impulsan la riqueza de un país se transfieren a través de la educación, a su vez, los recursos que se emplean para la educación de las nuevas generaciones son determinados por las capacidades de las diferentes capas sociales y, al reconocer que existen conjuntos de individuos más vulnerables, recomienda la intervención gubernamental. La existencia de clases sociales diversas sugiere que las desigualdades en el nivel de ingreso y los problemas de concentración de la riqueza, se revertirían si aumentaba la capacidad productiva de la economía, para tal efecto, la inversión estatal en educación, implicaba una más uniforme división del trabajo, a la par de una preocupación trascendente de política pública.

La educación de las clases bajas requiere acaso más atención en una sociedad civilizada, que la de las personas de cierta jerarquía y fortuna. Esta clase de gente cuenta más de dieciocho o veinte años de edad cuando abrazan cualquier profesión, actividad o industria, en la que piensan distinguirse. Entre tanto, disponen de todo el tiempo suficiente para adquirir, o hallarse en condiciones de perfeccionar más tarde, los conocimientos que los hagan dignos de la estimación pública o los capaciten para ello (Smith, 1776: 689).

Uno de los factores claves para el desarrollo de la política pública estriba en la educación, concebida no solo como la capacitación o la formación de individuos para el mercado laboral, sino bajo la connotación Kantiana que permita a la humanidad alcanzar el mayor grado de desarrollo que le sea posible.

"Únicamente por la educación el hombre puede llegar a ser hombre."

Immanuel Kant

De hecho, la educación presenta tal grado de relevancia en las tesis de Smith (1759) que se apoya en Hobbes y Mandeville para considerar que, la inevitable propensión humana hacia el egoísmo requiere de una comprensión ética y moral en los individuos para hacer posible una mejor convivencia social.

Pese a la visión optimista que Smith consignó en la Riqueza de las naciones, David Ricardo (1817) señaló de forma menos favorable uno de los problemas que debía atender la economía política, en relación con la distribución de los recursos, convirtiéndose en un nuevo desafío en materia de política pública: 
Toda la sociedad disfruta de la misma cantidad de agua y la misma cantidad de bienes, pero se distribuyen de modo diferente. Esto es así, sin embargo, suponiendo un monopolio del agua más que una escasez de ella. Si fuera escasa, la riqueza del país y la de los individuos disminuiría realmente en tanto se les privaría de una parte de sus satisfacciones. No solamente tendría el agricultor menos cereal para intercambiar por otros bienes necesarios o deseables para él, sino que a él y a cualquier otro individuo se les recorta el disfrute de una de las cosas más necesarias; no sólo habría una diferente distribución de la riqueza, sino una pérdida real de ella (Ricardo, 1817: 207).

A partir del problema de la distribución, el debate sobre la autosuficiencia del mercado para dirigir el mundo globalizado hacia una senda de convergencia en el ingreso per cápita o, si la intervención del Estado constituye, en su lugar, el camino más expedito; se ha extendido por multitud de enfoques y autores desde los clásicos hasta los contemporáneos. En particular, la escuela neoclásica se ha constituido en el escenario para el desarrollo de una teoría subjetiva del valor basada en el utilitarismo, donde el problema de política pública pretende ser resuelto a partir de la eficiencia en el sentido de Pareto.

El análisis de las implicaciones éticas en el contexto de la política pública promueve varios desafíos en materia moral para los individuos y para la sociedad a saber, V.gr., la forma en que se priorizan las demandas sociales y las individuales, la influencia de las últimas sobre las primeras y el efecto de las primeras en las segundas, los conflictos de interés cuando las preferencias individuales no se ajustan a las globales. Sobre este predicamento, Kaldor (1939) propuso una compensación para la capa social no beneficiada, basada en desarrollar mecanismos de transferencia monetaria que permitan mitigar las pérdidas de quienes hayan resultado afectados por el proceso de votación.

No obstante, los denominados mecanismos de compensación propuestos por Kaldor (1939) exigen que el desarrollo de la política gubernamental sea eficiente y transparente; el mundo que Arrow deseaba estudiar, donde se examinaba la posibilidad de que el bienestar social fuese el producto de juicios valorativos impersonales en cada individuo, exigía una consciencia moral y ética, tal como la que proponía Adam Smith frente a su definición de simpatía, el abandono de una postura egoísta y la preocupación por el otro; un mundo que en definitiva puede ser descrito mediante la 
retórica o la instrumentalización matemática, pero que difícilmente puede ser llevada a la práctica. Las consecuencias se hacen evidentes en el pequeño poema de Shantideva (Citado en Max-Neef y Smith, 2011):

"Todo el deleite que el mundo contiene proviene de desear la felicidad para los demás. Toda la miseria que el mundo contiene proviene de desear el placer para uno mismo"

\section{Utilitarismo como enfoque del Bienestar}

Consciente de las limitaciones de las instituciones, Bentham (1786) planteó que el legislador debía maximizar los beneficios de la sociedad al tiempo que debía minimizar sus perjuicios, lo que implica racionalizar los recursos con que el Estado cuenta a fin de ser eficiente ${ }^{3}$. Posibilitar un punto de bienestar social que fuese el resultado de una situación donde se lograsen conciliar las preferencias individuales con las colectivas; sobre esa base, la lectura ortodoxa pretendió que el mercado se constituía en el mecanismo social que exaltaba la capacidad de los seres humanos para, a través del intercambio, se aproximen a una situación de bienestar.

Bentham (1786), bajo el influjo del hedonismo de Epicuro y los postulados de Gossen frente a que el objeto de la vida humana reside en la maximización del placer y la minimización del dolor, trabajó en las primeras funciones de utilidad como una expresión cuantitativa de un postulado que exaltaría la racionalidad humana, al punto de señalar que el estándar de comportamiento de un individuo en la actividad económica, se reducía a maximizar su utilidad, satisfacción o beneficio minimizando el gasto o costo asociado. ${ }^{4}$

Cabe preguntarse, ¿Es posible modelar las consideraciones individuales y encontrar resultados sistemáticos cuando se trata de comprender el bienestar colectivo?, ¿Son las herramientas matemáticas tradicionales el camino más efectivo para estudiar los deseos individuales y las

\footnotetext{
${ }^{3}$ El propio Keynes señala que la postura de Bentham se basaba en un extremado individualismo. "No es contrario a la razón preferir la destrucción del mundo entero a un arañazo de mi dedo. No es contrario a la razón escoger para mí la ruina total para evitar la más pequeña incomodidad de [...] una persona totalmente desconocida para mí... la razón es y sólo debe ser la esclava de las pasiones y no puede pretender nunca otra tarea que servirlas y obedecerlas" (Bentham citado en Keynes, 1926: 65).

${ }^{4}$ Una reflexión a propósito, reside en determinar hasta qué punto el uso de los instrumentos técnicos, en sí mismos, constituyen herramientas definitivas para explicar la complejidad del comportamiento humano.
} 
necesidades colectivas?, ¿Es posible considerar resultados generalizables y susceptibles de réplica? ${ }^{5}$

En este preciso aspecto Arrow y Scitovsky (1974) señalaron un conflicto de intereses que no se resuelve, al menos desde la perspectiva tradicional. Las decisiones que competen a los designios de toda una sociedad, generalmente son asumidas por un número reducido de individuos. Para los autores, el utilitarismo ha sido incapaz de llevar a la práctica una situación de bienestar Paretiano, muy por el contrario, acrecientan los problemas de distribución y concentración de la riqueza evidenciados antes por Ricardo (1817) ya que, implicaban que el mercado no era capaz de asegurar el bienestar de la sociedad, ni las instituciones podían corregir las fallas del mismo; mientras que Edgeworth (1881) insistía en que el problema de la distribución podía resolverse mediante el encuentro de dotaciones factoriales eficientes y la dinámica del mercado.

Arrow (1974) sostuvo que la génesis de las dificultades en esta materia, se soportaban en las funciones de utilidad microeconómicas a través de las cuales, era posible la elección de una canasta de bienes que maximizaran el bienestar individual al mínimo costo o gasto, de manera que no constituían una suma agregada que redundara como producto en una función de bienestar global, que creciera como consecuencia de las funciones individuales, dado que, las preferencias de un individuo difieren significativamente de una persona a otra. Este problema constituía la agenda más trascendente de la teoría económica en el campo del bienestar:

If we continue the traditional identification of rationality with maximization of some sort, then the problem of achieving a social maximum derived from individual desires is precisely the problem which has been central to the field of welfare economics (Arrow, 1951: 329).

\footnotetext{
${ }^{5}$ A propósito, la crítica de Manfred Max-Neef, frente a los desarrollos de la ciencia económica y en particular al extremo uso de los instrumentos matemáticos como un fin en sí mismos, señala lo relevante de la ética y la filosofía moral que, se constituyen en la fuente del análisis económico: "La economía que originalmente se derivó de la filosofía moral, perdió de pronto gran parte de su dimensión humana que fue reemplazada por teorías caprichosas y trivialidades técnicas, incomprensibles para la mayoría e inútiles para todos, excepto tal vez para sus autores que suelen ganar premios por haberlas elaborado". (Max-Neef, 1986: 23).
} 
Por otro lado, la escuela neoclásica manifestaba que a partir de la modelación matemática, el equilibrio general era factible y que, además producía el máximo nivel de bienestar colectivo ${ }^{6}$, la advertencia de Arrow sobre una función de bienestar conjunta no se precisaba en una combinación de cestas de bienes y servicios, sino, en un conjunto de valores interpersonales. En consecuencia, la definición de un nivel de bienestar social dependía del establecimiento de dichos valores sociales.

In general, there will, then, be a difference between the ordering of social states according to the direct consumption of the individual and the ordering when the individual adds his general standards of equity. We may refer to the former ordering as reflecting the tastes of the individual and the latter as reflecting his values (Arrow, 1951: 333).

Tanto la función de bienestar social de un individuo como su función de utilidad particular, describen preferencias individuales, no obstante, en el caso de la primera, Arrow (1951) se basaba en consideraciones sociales impersonales que eran de tipo ético bajo un juicio imparcial, mientras que, en el caso de la segunda describía intereses personales o subjetivos que implicaban consideraciones egoístas y/o altruistas.

La postura de Arrow sobre el bienestar soportada en la ética promueve un examen más minucioso de la consciencia humana, precisando un enfoque superior que reemplaza los simples deseos o gustos como propensiones caprichosas o prejuiciosas frente al bienestar y bosquejan en su lugar, una búsqueda por aquellos valores objetivos que definan el modo de vida de una sociedad en su conjunto.

Debemos examinar todo el sistema de valores, cuando busquemos una teoría del bienestar social 'verdaderamente' general. El ordenamiento de acuerdo con los valores, es el que toma todos los deseos del individuo, incluyendo los deseos, sumamente importantes de

\footnotetext{
${ }^{6}$ En la visión neoclásica se asume que es posible encontrar una economía en situación de equilibrio, si los individuos actúan conforme a sus propios intereses entonces, el bienestar general se da por cosa dada; en efecto, intrínsecamente se sugiere que, si el equilibrio microeconómico existe, en consecuencia, existe también el equilibrio macroeconómico. Si se abandonaran por un instante las condiciones de método que dividen a la economía entre lo micro y lo macro, y se adoptará una posición de estudio de la economía como un todo, se advertiría, además, que no existe, en términos prácticos, un ejemplo de una economía en equilibrio y mucho menos de un individuo en la misma situación. Muy por el contrario, en cuanto a lo positivista se refiere, los seres humanos son impredecibles y complejos y, tanto sus conductas como sus decisiones difícilmente redundarán en el armonioso equilibrio colectivo que se pretende como cierto a pesar de no contar con la evidencia empírica y científica suficiente.
} 
carácter social, y es el que resulta primordialmente aplicable para la obtención de un máximo social. En cambio, el mecanismo del mercado sólo toma en cuenta el ordenamiento de acuerdo con los gustos (Arrow, 1950: 196).

Arrow (1951) indica que la única forma en que el tránsito de los gustos individuales a las preferencias sociales era posible cuando, involucraba la existencia de las dictaduras. Además, considerando que el enfoque neoclásico se caracteriza por la presencia de una psicología hedonista y filosofía utilitaria, asume que el bienestar de un individuo es idéntico a sus deseos, por lo que, en apariencia, el bienestar colectivo surge como la combinación de los deseos individuales, razón por la cual, en una economía de mercado se pretende la libertad para elegir los bienes de consumo ${ }^{7}$.

El hecho de que los supuestos puramente individualistas no puedan conducir a una función de bienestar social bien definida, significa, en efecto, que debe existir una divergencia entre los beneficios sociales y privados, para que podamos examinar un óptimo social. Una parte del sistema de valores de cada individuo debe ser una lista de normas socio-éticas, cuya realización no se puede lograr, por su misma naturaleza, mediante el comportamiento atomístico del mercado (Arrow, 1950: 210).

Con el precedente anterior, Arrow (1951) establece que existe un problema ético en la conducción de intereses individuales a máximos sociales, puesto que, los gustos implican preferencias individuales que se anteponen al bienestar general, exhibiendo que el mercado no resuelve de forma satisfactoria las fricciones entre los beneficios sociales y privados. Keynes (1926) recalca con mayor crudeza el hecho de que, de acuerdo con el análisis convencional, la creencia de que el bienestar colectivo no es sino la suma del bienestar individual constituye una presunción falsa.

El mundo no se gobierna desde arriba, de manera que no siempre coinciden el interés privado con el social. No es dirigido aquí debajo de manera que coincidan en la práctica. No es una deducción correcta de los principios de la economía que el interés propio

\footnotetext{
${ }^{7}$ Otro punto crítico aquí estriba en la creencia de que los individuos no se equivocan, o que sus curvas de aprendizaje permiten que a lo largo de un periodo de tiempo sus errores sean mínimos, o en términos técnicos, que las varianzas de las perturbaciones estocásticas sean pequeñas y constantes; pero la impredecibilidad de lo humano no sugiere que las personas asuman un comportamiento mecanicista. Tómese como ejemplo el film tiempos modernos de Charles Chaplin de 1936.
} 
ilustrado produzca siempre el interés público. Ni es verdad que el interés propio sea generalmente ilustrado, más a menudo, los individuos que actúan por separado persiguiendo sus propios fines son demasiado ignorantes o demasiado débiles incluso para alcanzar estos. La experiencia no demuestra que los individuos, cuando forman una unidad social, sean siempre menos clarividentes que cuando actúan por separado (Keynes, 1926: $81)$.

Precisamente, el problema al que se enfrenta Arrow, reside en estudiar la posibilidad de que un máximo social surja como respuesta a los deseos particulares de los individuos, siendo todos tan disimiles e inconstantes; no obstante, en ese tránsito, el autor se tropieza con el incumplimiento de la condición de racionalidad, sumada a la dificultad de un óptimo de Pareto puesto que, la decisión tomada por una sociedad basada en un régimen democrático, a partir de un proceso de votación, donde la mayoría decide, produce como resultado que la minoría no satisface sus demandas y, el máximo social no abarca a la sociedad por completo (Arrow, 1950).

Y en el mismo sentido, Keynes (1926) subraya que el trabajo de Marshall da cuenta de la fragilidad del concepto del laissez-faire, dado que existen casos donde el interés social y el interés privado eran diferentes. En consecuencia, para Arrow los mecanismos del mercado no resuelven los conflictos derivados de las preferencias individuales y el bienestar social, a menos que se asuma una óptica alternativa al modelo convencional, como se manifiesta en la propuesta de Sen sobre una disrupción a las dinámicas de mercado que, implican una ruptura frente al utilitarismo de Bentham.

Otra de las dificultades de la postura neoclásica, radica en el hecho de deducir que el bienestar humano puede definirse y limitarse en términos monetarios o el acceso a bienes materiales de consumo, considerar al mismo tiempo, que la economía es una ciencia totalitaria y suficiente para dar respuestas a los problemas sociales, una suerte de miopía teórica que la escuela neoclásica hizo evidente cuando, a partir, de las premisas de Bentham, aceptó que la felicidad humana equivalía a una función de maximización de la utilidad derivada del consumo de bienes materiales. Pronto el tipo de sociedad que se preocupaba por la producción de aquello necesario para la vida, se transformó en un tipo de mercado que, en su lugar, creaba necesidades donde antes no las había. A propósito de esto, en 1996, el novelista Chuck Palahniuk escribió lo siguiente: 
Hay un tipo de mujeres y de hombres jóvenes y fuertes que quieren dar sus vidas por una causa. La publicidad hace que compren ropas y coches que no necesitan. Generaciones y generaciones han desempeñado trabajos que odiaban para poder comprar cosas que en realidad no necesitaban (Palahniuk, 1996: 72).

Arrow enuncia que existe una imposibilidad manifiesta para el desarrollo de política pública en una economía regulada por el mercado, dadas las inconsistencias en los procesos electorales y los intereses antagónicos entre los mismos individuos de una sociedad que, se basa en las preferencias y no en la definición valorativa de determinantes del bienestar colectivo. De la misma forma MaxNeef (1986) exhorta que, el concepto de utilidad en el que, actualmente se basa la economía moderna, hace abstracción de los multitudinarios factores que asisten a la explicación del bienestar reduciéndolos a una única cosa: aquello que poseemos.

El panorama desolador que Arrow avizoró cuando sorteó las fronteras de su teorema de imposibilidad trasciende, desde luego, no únicamente a las limitaciones de una simbología matemática, los teoremas o axiomas de la lógica económica, porque aborda una cuestión ética más relevante, y es que, por mucho que se pretenda denominar el tipo de instrumento social: mercado en algunos escenarios o intervención estatal en otros, según sea conveniente, el problema reside en la comprensión de las complejidades propias de la conducta humana y en que, siendo miembros de una misma especie, existan dificultades para promover la solidaridad y el altruismo.

\section{Perspectiva de Sen y Deaton sobre el Bienestar}

En respuesta a los estudios realizados por Bentham, Marshall, Jevons, Mill, Pigou y otros, respecto a la trascendencia de la utilidad en relación al bienestar, Amartya Sen, efectúa una crítica a postulados welfaristas y consecuencialistas del utilitarismo como pilar de un óptimo social.

Sen en consonancia con Arrow, señala que, por medio de la utilidad no es posible realizar comparaciones valorativas que inviten al entendimiento del bienestar colectivo, dado que no permiten "realizar ordenaciones basadas en la suma, que sí requieren comparaciones interpersonales" (Sen, 2000: 82-83), toda vez que, estudia a cada agente desde su elección individual, implicando imprecisión a la hora de determinar sus preferencias individuales. 
La perspectiva utilitaria, para el autor se caracteriza por contar con dificultades prácticas para cada individuo que desea adherir a una particular función de utilidad, dependiendo de factores personales o situacionales. Sen planeta que ciertos agentes son susceptibles de generar preferencias suntuosas en comparación con sus ingresos, razón por la cual, el nivel de bienestar asociado se restringe. En consecuencia, se desarrolla una tendencia hacia la indiferencia en la distribución de la desigualdad, que asiste de forma restrictiva los cálculos para aquellos que sufren de situaciones adversas y de privaciones.

Para Sen (2000) las divergencias existentes en las preferencias y selecciones individuales, se restringen al acto comparativo interpersonal cuando se abordan desde una postura ortodoxa, dadas por ejemplo, características predominantes en poblaciones vulnerables, particularmente objeto de privaciones mediante violaciones a sus derechos y libertades.

Para el autor la teoría neoclásica se limita "simplemente a la felicidad o el placer y a veces la satisfacción de deseos hacia las medidas de placer o felicidad" (Sen, 2000: 90), con el propósito de identificar a los individuos con mayor o menor nivel de bienestar, partiendo de una medida mental, y por ende, subjetiva, en las elecciones de los mismos, haciendo que tales transgresiones sean consideradas indirectamente dentro de dicha medición. Adicionalmente, sugiere que es fundamental dar cumplimiento y respeto a derechos de los agentes que componen la economía, por medio de factores como "la educación básica, asistencia sanitaria elemental y la seguridad en el empleo" (Sen, 2000: 86), que permitan el bienestar de cada uno en su entorno.

En segundo lugar, Sen afirma que la utilidad como instrumento de medición del bienestar se vincula tajantemente al consumo, condicionándolo hacia la mera satisfacción del individuo, dejando el precedente de elevar la utilidad a futuro con nuevas canastas de bienes o servicios que así lo permitan.

Este aspecto crítico es compartido por Manfred Max-Neef (1986), pues manifiesta que la industria mundial, en especial, aquella que pertenece a los países desarrollados, incita al consumo voraz de productos que se promueven como imprescindibles para la vida, con el propósito de sentir mayor "bienestar", haciendo que se vinculen las necesidades hacia factores externos, fundamentalmente. 
La dependencia consumista en la que se encuentra inmersa la sociedad, se amplía hacia la imitación de ideales, cultura, pensamiento, modelos legales y económicos, causando que los países en vías de desarrollo, acaten los lineamientos de aquellos que tienen relativa "superioridad", motivo por el cual Max-Neef et al., (1986: 85) planean que "los países pobres están sometidos al arbitrio de los países ricos, y a1 interior de los países pobres sucede lo mismo que entre países pobres y ricos". Sen y Williams advierten que el utilitarismo concibe a las personas como entidades que únicamente pueden desear, tener placer y sentir dolor; puesto que, una vez que se ha tomado nota de la utilidad de la persona, ya no es un ser imprescindible (Sen \& Williams, 1977). Por tanto, esta vertiente de pensamiento abandona el postulado de multidimensionalidad intrínseca del ser humano, como dinamizador de su contexto en ámbitos básicos en los que debe participar para el fortalecimiento de su desarrollo integral.

Sen (2000) propone su teoría de las libertades fundamentales o capacidades, y, de los funcionamientos; donde a grandes rasgos, profundiza el hecho de procurar mayor atención a las oportunidades reales que cada individuo puede lograr a partir de su ser y hacer. En su conceptualización de las funciones, el autor se cimenta en la valoración que cada uno ofrece a aquello que pueda realizar, desde lo elemental, entendido como los estados alcanzados a causa de una buena alimentación y el no padecimiento de enfermedades (Sen, 2000); hasta lo complejo, incluyendo la capacidad de participación democrática y desenvolvimiento social y, la concepción de respeto hacia el propio ser. Por tal motivo, el individuo no evalúa lo que tiene materialmente, como se hace en la base teórica del utilitarismo, sino que, da valor a lo que puede alcanzar por medio de ese bien.

En complemento, las capacidades se refieren a la combinación de las funciones que la persona puede alcanzar, eligiendo diferentes alternativas, que se ajusten a sus objetivos primordiales (Sen, 2000), por consiguiente, el autor propone una nueva visión del concepto de libertad.

El direccionamiento que Sen proporciona a la teoría del bienestar, se sumerge en la aceptación de las diferencias que cada ser humano tiene, para el alcance de mejores condiciones de vida. Por lo cual, ofrece énfasis y total aprecio por la heterogeneidad en las evaluaciones que realiza frente a sus funciones y, en consecuencia, sus capacidades, puesto que, en contraste al utilitarismo, no busca sosegar las diferencias hacia la agregación de magnitudes, sino que afirma que a los agentes 
les corresponde insistir en que, de solo haber una magnitud homogénea para valorar, se reduce de manera radical el campo del razonamiento valorativo (Sen, 2000).

Por tanto, dentro del enfoque de Amartya Sen, no se presentan las dificultades en las comparaciones interpersonales vistas desde la utilidad, ya que, por medio de las funciones, se abre la posibilidad de encontrar un sinfín de medios cambiantes que posibiliten el cumplimiento de diversos objetivos, sin estar sumidos en el condicionamiento mental que evocan autores como Bentham.

La variabilidad de la conversión de los medios en fines o en la libertad para alcanzar esos fines se refleja en la magnitud de esos logros y libertades que pueden figurar en la lista de fines. Estas son las ventajas de utilizar la perspectiva de las capacidades para realizar evaluaciones y valoraciones (Sen, 2000: 101).

Cabe aclarar que, Sen (2000) declara que dentro de su teoría, es imprescindible ponderar el peso que se concede a la libertad fundamental frente al logro real, pues no todos los aspectos que una persona considere importantes en una situación, lo serán para otra. Gallego (2015), resume de manera clara, el enfoque de capacidades de Sen, enunciando tres premisas principales:

a) el bienestar debe ser buscado como un fin, dando trascendencia no solo a los bienes sino además a los derechos $\mathrm{y},(\ldots)$ a una teoría de justicia universalista que tenga como objetivo la expansión de las capacidades y la superación de un umbral mínimo de capacidades; b) la consideración del bienestar como un estado intrínseco de la persona, resaltando la heterogeneidad personal y por ende, la capacidad diferenciada de cada persona para lograr un bienestar; c) la ruptura de los supuestos de la economía tradicional (...), ya que estos disuelven la posibilidad de abordar cuestiones de género, infancia, distribución de libertades al interior de la familia y temas que van más allá de la falta de bienes (Gallego, 2015: 59).

Así, la teoría de Sen respecto al bienestar, se vincula a la amplitud del entendimiento de los individuos dentro de su aspecto singular y como parte de un ente comunitario y social, enfocándose en las diferentes variables y situaciones que contempla en su vida cotidiana como es el caso de su 
salud, empleo, vida social y política, haciendo que el consumo quede relegado como una parte de la universalidad del ser en sí mismo.

Dentro de esta línea de ideas, la teoría desarrollada por Angus Deaton ${ }^{8}$ (2015) se refiere a un concepto de bienestar amplio, que dilucida la complejidad de la vida humana desde sus necesidades de consumo, mediante la riqueza y el ingreso; factores físicos o psicológicos, estudiados a partir de la calidad de la salud y su felicidad, así como del nivel educativo del individuo y su habilidad para participar en la sociedad civil por medio de instancias como la democracia y los estamentos de Ley que tenga cada país.

Deaton realiza un discernimiento de los factores que implicarían para un agente tener bienestar puesto que, existen dicotomías propias entre cada individuo y las oportunidades a las que tenga acceso. Hace alusión a una visión objetiva, desarrollada por Sen, en la que se relaciona una subsistencia en procura de "una esperanza de vida prolongada, buena salud, ausencia de pobreza, democracia e imperio de la ley" (Deaton, 2015: 45).

No obstante, para el autor es trascendental matizar este concepto con connotaciones subjetivas, aunque no en el mismo sentido de los teóricos utilitaristas, pues busca entender el nivel de felicidad, a partir de la percepción individual de las personas, dado que, estos sentimientos contribuyen a la consecución de una vida digna. Por este motivo, concibe al bienestar como "todas las cosas buenas para una persona, que hacen que la vida sea buena" (Deaton, 2015: 44).

El punto de partida del pensamiento de Deaton frente a la política económica, comprende la trascendencia de la heterogeneidad dentro del concepto de bienestar, pobreza e incluso el desarrollo económico, por cuanto sugiere que el papel de la individualidad en las decisiones de consumo y/o ahorro de los agentes que conforman una economía es decisiva para el buen vivir, dependiendo de los niveles de ingreso que puedan conseguir. Sus estudios alrededor del ingreso lo incitaron a proponer metodologías alternativas en relación a la obtención de datos y su interpretación.

\footnotetext{
${ }^{8}$ Premio Nobel de Economía año 2015.
} 
La propuesta de Deaton entorno a las medidas de diferentes variables económicas, parte de instancias individuales (enfocadas en lo micro) que pueden contemplar interacciones agregadas (orientación macro), razón por la cual, promulga el uso de encuestas al núcleo familiar, puesto que permiten reconocer el comportamiento de los individuos respecto al uso del ingreso en los diversos bienes existentes, posibilitando identificar las diferencias comportamentales entre sectores de personas con mayores comodidades, de aquellas más desfavorecidas.

El bienestar comprende una amplia interacción de variables, con diferencias y particularidades inherentes a cada nación, ejemplo de ello, manifiesta Deaton (2015) son los países con mayor ingreso per cápita, ya que poseen mejores sistemas de salud, que impulsan una esperanza de vida superior, una menor morbilidad y mortalidad, conllevando a una vida más feliz, en contraste con contextos sociales donde las limitaciones juegan en contra de la obtención del bienestar. En conjunto, expone que aquellos países con mejoras en las tasas de educación en sus diferentes niveles, especialmente en los superiores, ejercen repercusiones favorables tanto en salud, como en lo financiero.

\section{CONCLUSIONES}

El abordaje teórico que el bienestar ha suscitado dentro de la comunidad académica, ha propiciado un debate interesante a raíz de las discrepancias conceptuales de los autores referidos; sin embargo, el propósito real de los discernimientos es enriquecer las posturas existentes, por medio de la crítica o la complementación, pues a partir de ellos se pueden formular, proponer y ejecutar planes, programas y políticas públicas a diversos territorios, dependiendo de sus necesidades y problemáticas.

La posición subjetiva del bienestar manifiesta la importancia de los conceptos valorativos individuales, frente a los bienes que procuren la maximización de su situación económica. A través de elaboradas formulaciones matemáticas en función de la utilidad, se pretende encontrar el punto óptimo, en términos Paretianos, que logre una distribución de recursos conveniente, en función de una mejor calidad de vida.

El enfoque de Sen, ofrece una visión del bienestar que no se fundamenta en la valoración del bien materialmente, sino por el contrario, valora aquello que se puede lograr por medio de él, 
especialmente en relación a mejorar la esperanza de vida, alcanzar altos niveles educativos y mantener un buen estado de salud.

La política pública dentro de su ejercer social y comunitario, debe efectuar estrategias que conduzcan al bienestar, entendiendo que éste depende de diversas fuentes, con el propósito de llegar a situaciones de buen vivir. El acceso a salud, educación y oportunidades de empleo, son fundamentales para forjar situaciones adecuadas que contribuyan al desarrollo integral de la sociedad.

Finalmente, cabe precisar que el estudio del bienestar, debe ligarse a fenómenos como la pobreza y miseria, la desigualdad, el crecimiento y desarrollo económico, dado que es menester la búsqueda de los problemas base, con el objetivo de plasmar posibles soluciones que permitan una vida de calidad apropiada, desde el punto de vista objetivo como subjetivo, entrelazando el quehacer individual con el colectivo y el Estatal.

"No es de esperarse que los reyes filosofen o que los filósofos se conviertan en reyes; tampoco es algo deseable, pues la posesión del poder ineludiblemente corrompe el juicio puro de la razón". Immanuel Kant.

\section{REFERENCIAS}

(1) Arrow, K. (1951). Social Choice and Individual Values. John Wiley y Sons, Inc.

(2) Arrow, K., \& Scitovsky, T. (1974). La Economía Del Bienestar. Fondo de Cultura Económica.

(3) Bentham, J. (1786). Filosofía de la ciencia económica. Fondo de Cultura Económica.

(4) Deaton, A. (2015). El gran escape. Salud, riqueza y los orígenes de la desigualdad. Fondo de Cultura Económica.

(5) Edgewoth, F. (1881). Mathematical Psychics: An essay on the aplication of mathematics to the moral science. Stanford University Libraries.

(6) Gallego, L. (2015). Bienestar y género bajo el enfoque de las capacidades. Aplicación al caso de Medellín [Universidad Autónoma de Barcelona]. https://www.tdx.cat/bitstream/handle/10803/298332/lmgd1de1.pdf?sequence=1 yisAllowed=y

(7) González, J. (1998). Arrow: la elección, los valores y la ideología de mercado. Cuadernos de Economía, 17(28), 27-41. https://revistas.unal.edu.co/index.php/ceconomia/article/viewFile/11609/20646 
(8) Kaldor, N. (1939). Welfare Propositions in Economic. The Economic Journal, 49(195), 549-552. https://doi.org/https://doi.org/10.2307/2224835

(9) Keynes, J. (1926). The end of the Laissez-faire. The economic consequences of the peace. Great Minds Series.

(10)Kitchenham, B. (2004). Procedures for Performing Systematic Reviews. Keele University. http://www.inf.ufsc.br/ aldo.vw/kitchenham.pdf

(11) Max-Neef, M. (1986). La economía descalza: Señales desde el mundo invisible. Nordam Comunidad.

(12) Max-Neef, M., Elizalde, A., \& Hopenhayn, M. (1986). Desarrollo a Escala Humana. Conceptos, aplicaciones y algunas reflexiones (Tercera Ed). Icara Editorial.

(13) Max-Neef, M., \& Smith, P. (2011). La economía desenmascarada: del poder y la codicia a la compasión y el bien común. Editorial Icaria.

(14) Palahniuk, C. (1996). El club de la pelea. Editorial de Bolsillo.

(15) Petty, W. (1899). The Economic Writings of Sir William Petty. Cambridge University Press.

(16) Ricardo, D. (1817). Principios de Economía Política y Tributación. Fondo de Cultura Económica.

(17) Sen, A. (2000). Desarrollo y Libertad. Planeta.

(18) Sen, A., \& Williams, B. (1977). Utilitarism and beyond. Cambridge University Press.

(19) Smith, A. (1759). Teoría de los sentimientos morales. Fondo de Cultura Económica.

(20) Smith, A. (1776). Investigación sobre la naturaleza y causas de la riqueza de las naciones. Fondo de Cultura Económica. 